\title{
In-situ liquid cell TEM investigation on assembly and symmetry transformation of Pt superlattice
}

\author{
Junyu Zhang, Shi-Gang Sun and Hong-Gang Liao*
}

\begin{abstract}
Two dimensional (2D) nanocrystal functional superlattices with a well controlled structure are of significant importance in photonic, plasmonic and optoelectronic applications and have been well studied, but it remains challenging to understand the formation mechanism and development pathway of the superlattice. In this study, we employed in-situ liquid cell transmission electron microscopy to study the formation of $2 \mathrm{D}$ superlattice and its local phase transition from hexagonal-to-square nanocrystal ordering. When colloidal nanocrystals flowed in the solution, long-range ordered hexagonal superlattice could be formed either through shrinking and rearrangement of nanocrystal aggregates or via nanocrystal attachment. As the nanocrystals' shape transformed from truncated octahedral to cube, the local superlattice rearranged to square geometry. Moreover, our observations and quantitative analyses reveal that the phase transition from hexagonal to square mainly originates from the strong van der Waals interactions between the vertical (100) facets. The tracking of $2 \mathrm{D}$ cube superlattice formation in real-time could provide unique insights on the governing force of superlattice assembling and stabilization.
\end{abstract}

Keywords: shape transform, hexagonal-to-square, van der Waals interactions, liquid cell transmission electron microscopy

\section{INTRODUCTION}

Recently, two-dimensional (2D) superlattices with unique physical and chemical properties have triggered considerable research interests witnessed from photonic, plasmonic and optoelectronic applications [1-6]. In particular, the well-shaped 2D superlattices have been created by changing the sizes, shapes, surface chemistry of nanocrystals in solution [7-12]. However, understanding the self-assembly mechanisms of $2 \mathrm{D}$ superlattice was based on the "quench-and-look" approach through characterizing the initial colloidal state and final super- lattice state, or investigation by in-situ methods such as $\mathrm{X}$-ray diffraction, small-angle X-ray scattering, atomic force microscopy and scanning tunneling microscopy [13-15]. Though all of these methods have acquired important information on the self-assembly, they met a lot of experimental challenges on the direct visualization of the complicate and delicate dynamic processes at high spatial resolution.

With the technical advances in electron microscopy, liquid cell transmission electron microscopy (TEM) has acted as a direct and useful tool for observing material transformation in real-time at atomic resolution [16-21]. Nanocrystal superlattice formations through self-assembly in solution have been studied by liquid cell TEM [2226]. Park et al. [22] first reported that the capillary forces and local solvent evaporation could drive the randomly distributed nanocrystals into ordered nanocrystal superlattice. The lateral dragging of solvent boundaries is the major driving force for the formation of $2 \mathrm{D}$ superlattice near the liquid interface. Furthermore, Lee et al. [23] emphasized the solvent boundary primarily affected the formation of superlattice regardless of various conditions, including substrates, the types and the concentration of nanocrystals. Two pathways of the $2 \mathrm{D}$ superlattice selfassembly were elucidated: lateral dragging and local flattening. In addition, Chen's group [24] reported the electron beam induced structural modulation of superlattices in real-time. The size, shape and structure of the nanocrystals could have significant effects on the formation and stabilization of superlattices in solution. It is noted that all observations in previous work have used pre-synthesized nanocrystals as research targets, but the direct evidence of how the shape affects the self-assembly is rare [22-24,26-28]. Therefore, it is highly desirable to investigate the assembling process using transformable building blocks in real-time and real-space.

State Key Lab of Physical Chemistry of Solid Surfaces, Collaborative Innovation Center of Chemistry for Energy Materials, College of Chemistry and Chemical Engineering, Xiamen University, Xiamen 361005, China

* Corresponding author (email: hgliao@xmu.edu.cn) 
With this motivation, we visualized the $2 \mathrm{D}$ superlattice formation and its transformation in solution during nanocrystal shape changing. The $2 \mathrm{D}$ hexagonal superlattice formation was achieved first and the transition from ordered hexagonal structure to square structure along with shape evolution of nanocrystal building blocks was directly captured too, as shown in Fig. 1. The force analysis during the phase transformation of $2 \mathrm{D}$ superlattice revealed the origin of superlattice stabilization. It is anticipated that the present study could provide new insights on the rational design of $2 \mathrm{D}$ nanomaterials.

\section{EXPERIMENTAL SECTION}

\section{Materials}

All chemicals, including $\mathrm{Pt}(\text { acetylacetonate })_{2}\left(\mathrm{Pt}(\mathrm{acac})_{2}\right.$, 99\%, Aldrich), dimethyl formamide (DMF, Sinopharm Chemical Reagent Co., Ltd., Shanghai, China), potassium hydroxide (KOH, Sinopharm Chemical Reagent Co., Ltd., Shanghai, China), indium (In, 99.999\%, Aldrich), ultrathin silicon wafers $(100 \mu \mathrm{m}, 4$-inch, p-doped, Virginia Semiconductor) and acetone, were used as received

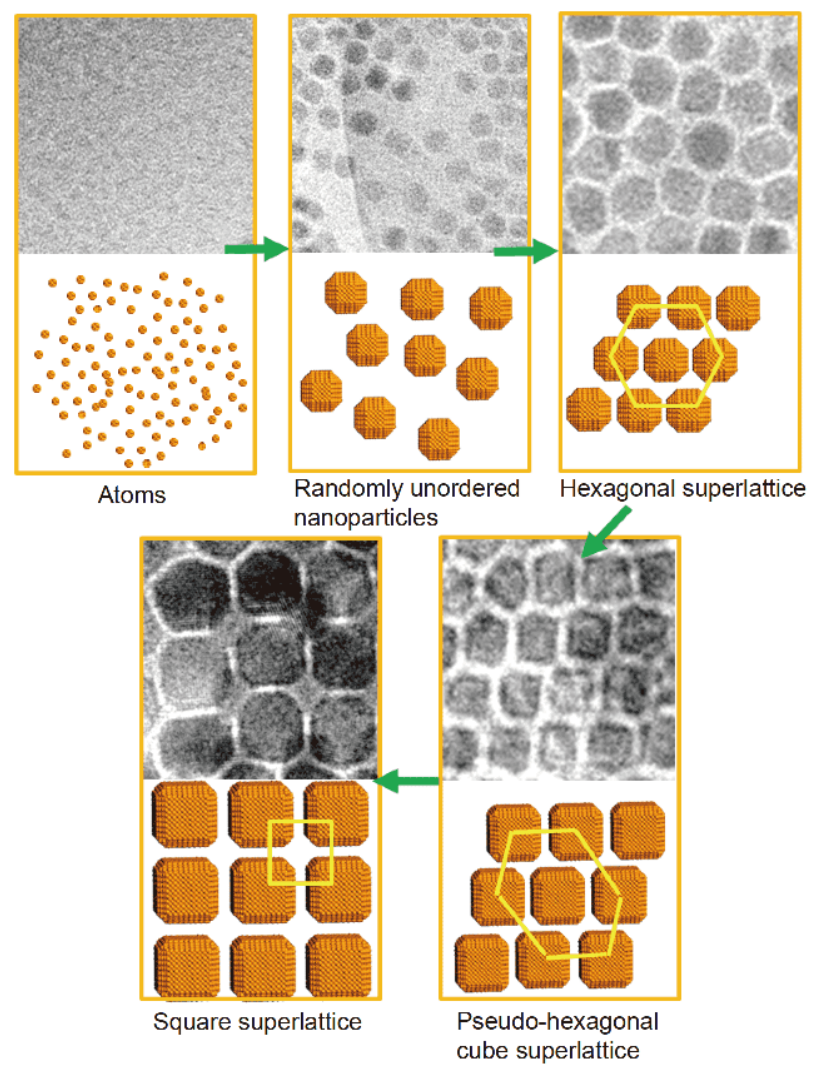

Figure 1 Schematic illustration of the formation and phase transition of 2D superlattice. without further purification.

\section{Synthesis of Pt cube}

The synthesis of Pt cube referred to the Ref. [29]. In a typical experiment, approximately $16 \mathrm{mg}$ of $\mathrm{Pt}(\mathrm{acac})_{2}$ was dissolved in $40 \mathrm{~mL}$ of DMF. The mixture was sealed inside a poly(tetrafluoroethylene) lined autoclave and maintained at $180^{\circ} \mathrm{C}$ for $48 \mathrm{~h}$. The reaction temperature by heating was at a rate of $10^{\circ} \mathrm{C} \mathrm{min}{ }^{-1}$. Finally, the thusobtained nanocrystals were re-suspended in alcohol for further TEM characterizations.

\section{Fabrication of liquid cell}

Liquid cells were made as described in previous publication with slight modifications [16,22]. Firstly, silicon nitride membranes with $15 \mathrm{~nm}$ thick were deposited on the surface of Si. After photolithographic patterning and etching, the bottom chips of the liquid cell were deposited with indium spacer of $100 \mathrm{~nm}$. Finally, the bottom and top chips of the liquid cell were aligned and boned.

\section{In-situ TEM imaging process}

The growth solution was prepared by dissolving $\mathrm{Pt}(\mathrm{acac})_{2}$ $\left(30 \mathrm{mg} \mathrm{mL}^{-1}\right.$ ) or pre-synthesized Pt cube in DMF. The solution was drawn into the cell by capillary force and formed a liquid layer $(100 \mathrm{~nm})$ sandwiched between two silicon nitride membranes at the window. The liquid cell was then loaded into the microscope as a standard TEM sample for imaging. FEI F20 TEM operated at $200 \mathrm{kV}$ and beam current densities of 427, 2250, 3320 and $5000 \mathrm{e} \AA^{-2} \mathrm{~s}^{-1}$ were maintained for this study. All movies were recorded at 5 frames per second using a Gatan SC832 camera (fiber optical charge-coupled device (CCD)).

\section{RESULTS AND DISCUSSION}

Movie S1 (Supplementary information) displays the growth and assembling of platinum nanocrystals into $2 \mathrm{D}$ hexagonal structure. Under the electron beam irradiation, many nanocrystals grew and rotated freely first. The nanocrystals aggregated and shrank laterally in solvent while the nanocrystal concentration increased. Finally, a hexagonal close-packed ordered structure was formed. The hexagonal patterned nanocrystals formed and were stabilized as shown in Fig. 2a (marked 0-6, 68 s). Moreover, Movie S2 shows the process of solvent-driven coalescence of two groups of ordered nanocrystals. One group with a small number of ordered nanocrystals moved to another and merged together into a larger hexagonal pattern as shown in Fig. S1. During this pro- 

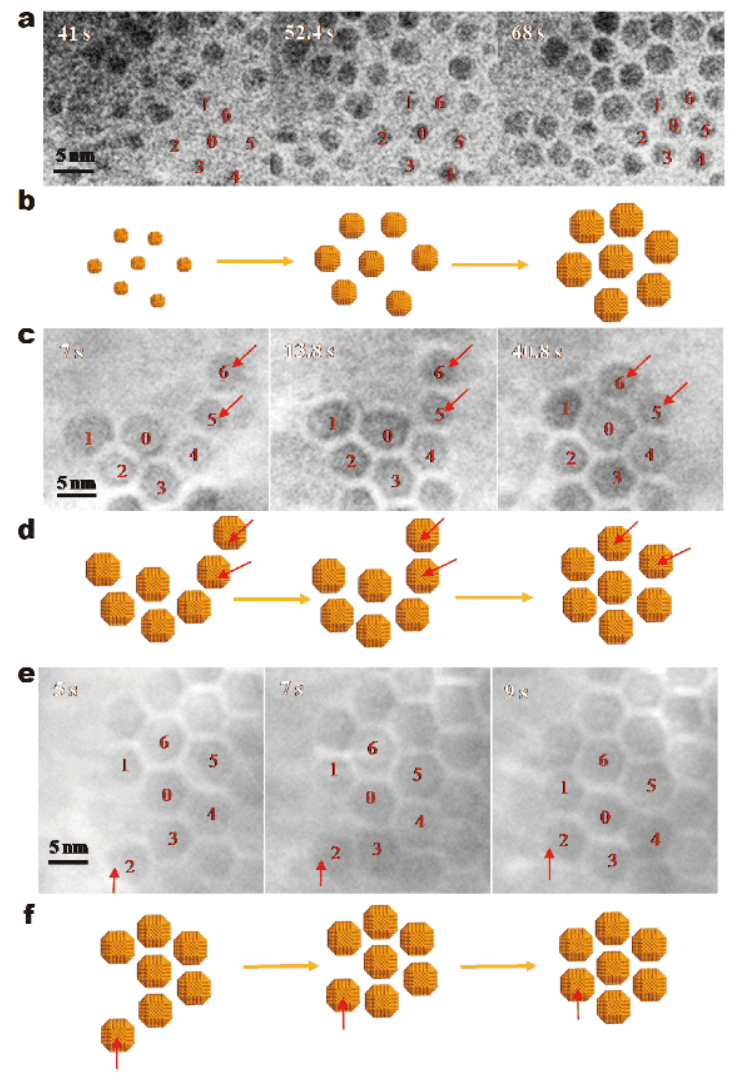

Figure 2 In-situ TEM imaging of the formation of hexagonal closepacked ordered structure. (a) Sequential TEM images showing the nucleation, growth and assembly of Pt close-packed ordered structure extracted from Movie S1; (b) the detailed flowchart of (a); (c) sequential TEM images showing the growth of the loosely packed hexagonal closepacked ordered structure with six coordination numbers by reordering the nanocrystal aggregate extracted from Movie S3; (d) the detailed flowchart of (c); (e) sequential TEM images showing single particle addition onto the domain of a close-packed ordered structure extracted from Movie S2; (f) the detailed flowchart of (e).

cess, a nanocrystal chain could spilt from one assembly and approach the opposite assembly like a "tentacle", which could be induced by the strong interaction between the assembly and liquid fluctuation. As the "tentacle" contacted with the opposite assembly (marked with a yellow arrow), it dragged two assemblies together and then the assembly shrank until all the vacancies were filled. Another hexagonal assembly formation has been imaged in Movie S3, close-packed assembly with six coordination numbers was also formed by particle aggregation and reordering. As illustrated in Fig. $2 \mathrm{c}$ and d, nanocrystals aggregated and formed chains at the beginning $(7 \mathrm{~s})$ due to the van der Waals interaction, and then the nanocrystal chains (marked 3-6) folded and formed loosely packed clumps (40.8 s). Fig. S2 shows the statistic of angle changes formed by three adjacent nanocrystals, where the angle (such as, 6-0-1) reduces significantly. The results suggested that the folding behavior occurred. Finally, the angles by any three adjacent nanocrystals were close to $60^{\circ}$, indicating the formation of perfect hexagonal symmetry. Additionally, the long-range ordered hexagonal superlattice can be also achieved via addition of single nanocrystals. As Fig. 3 displays (also seen in Movie S4), a burst of nucleation in solution happened at the beginning $(0 \mathrm{~s})$. Subsequently, large number of nanocrystals emerged under constant illumination from 0 to $32 \mathrm{~s}$. During this stage, nanocrystals nucleation, growth and self-assembly occurred in parallel. Later, individual nanocrystal incorporated into the existing superlattice, and filled the kink site to form an ideal close-packed hexagonal structure from 32 to $135 \mathrm{~s}$ (marked in the red circle and yellow arrow). These nanocrystals were moving around and got stationary on a certain site of the superlattice, due to the lower energy at

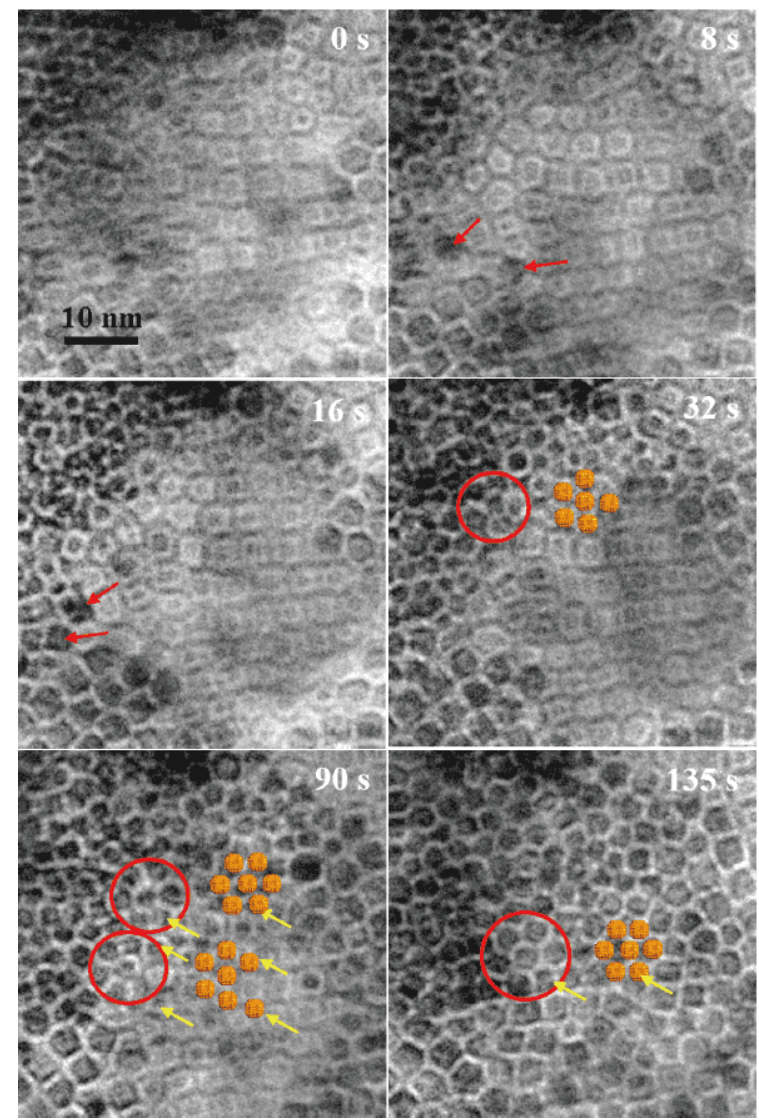

Figure 3 In-situ TEM imaging of the formation of hexagonal superlattice. Sequential TEM images showing the formation process of the long-range ordering hexagonal superlattice via site selective nanoparticle attachment method extracted from Movie S4. 
the kink site. As displayed in Fig. 2e and $\mathrm{f}$ (also seen in Movie S2), the single nanocrystal tended to move to the kink site (marked with a red arrow). Finally, the perfect hexagonal close-packed ordered superlattice was obtained. In a word, by either the shrinking, reordering of nanocrystal aggregates or nanoparticle attachment, longrange ordering hexagonal close-packed superlattice can be formed, which maximizes close-range van der Waals interactions in agreement with previous work $[22,23,25]$, demonstrating that many types of forces (such as, van der Waals force, Brownian force and steric hindrance force) drive the ordered hexagonal structure formation and nanoparticle self-assembly during the early stage.

As the growth proceeded, the hexagonal-to-square phase transition was observed. Fig. S3a shows the hexagonal-to-square phase transition process in a small area of ordered superlattice (also seen in Movie S5). At the beginning $(5 \mathrm{~s})$, the hexagonal close-packed truncated octahedra nanocrystals with the coordination number of six (marked number 1-6) were observed. As the particles grew, the truncated octahedra nanocrystals grew into nanocubes, and then the hexagonal superlattices transformed into disordered cubes at $120 \mathrm{~s}$. The disordered cubes (marked number 4-6) aligned with one of their [100] directions quickly (235 s). The van der Waals interactions between the two cubes with opposed $\{100\}$ facets led to reduced rotational freedom. Finally, the directionality between cube-to-cube in-plane (100) attractions changed the symmetry of the local superlattice from hexagonal to square (320 s). During the phase transition process, the change of angles of three adjacent nanocrystals was plotted in Fig. S3b. It is clear that all angles are almost $60^{\circ}$ (also see those marked in Fig. S3a, $5 \mathrm{~s}$ ), suggesting that the superlattice is hexagonal geometry at the early stage. At around $120 \mathrm{~s}$, the angles quickly changed, and all angles moved toward $45^{\circ}$ or $90^{\circ}$. At the later period, the angles continued to change slowly due to the confined space between particles. Finally, the angles were almost $90^{\circ}$ or $45^{\circ}(320 \mathrm{~s})$, the square packing cubes were achieved, and the rotations were fully prohibited at the end. The scheme in Fig. S4c depicts the detail process of hexagonal-to-square phase transition. Moreover, Movie S6 demonstrates the local phase transition process of a large nanoparticle assembly from hexagonal close-packed to square geometry. The typical particles extracted from Movie S6 were shown in Fig. 4a. The scheme in Fig. 4b depicts the detail process of hexagonal-to-square phase transition. The hexagonal close-packed symmetry was broken due to the shape change of nanocrystals, and then formed pseudo-hexagonal structure from 0 to $443 \mathrm{~s}$.
Fig. S4 shows the statistics of the angles' changes during the formation of square nanocube, where the angles gradually change from $60^{\circ}$ to $90^{\circ}$ or $45^{\circ}$ in a large nanoparticle assembly. Compared with the small assembly in Movie S5, the larger one needs more time to reach a new stable phase $(690 \mathrm{~s})$. In principle, the Brownian force is the main driving force of particle motion and selfassembly, while van der Waals attractive force $\left(F_{\mathrm{vdW}}\right)$ imposes an attractive force between nanoparticles at relatively short particle separations. During the phase transition process, the distance between nanoparticles is relatively short, and thus van der Waals attractive force is the dominant driving force for the assembly process $[25,26]$. Thus, the real-time van der Waals attractive force between individual particles was calculated in the system:

$F_{\mathrm{vdW}}(d, D)=-\frac{A}{3}\left[\frac{d^{2} D}{2\left(D^{2}-d^{2}\right)^{2}}+\frac{d^{2} D}{2 D^{4}}-\frac{D}{D^{2}-2 d^{2}}+\frac{1}{D}\right]$,

where $d$ is the diameter of Pt nanoparticle (Fig. 4c), $D$ is the particle center-to-center distance (Fig. $4 \mathrm{~d}$ ), and $A$ is Hamaker constant for Pt in DMF. As the shape changed to cube after $225 \mathrm{~s}, F_{\mathrm{vdw}}$ continued to increase, and reached its maximum about $20.2 \times 10^{-12} \mathrm{~N}$ as shown in Fig. 4e. And many well-ordered square phase superlattice domains in the 2D film are displayed in Fig. S5 (marked by circles). The corresponding energy-dispersive X-ray spectroscopy data confirm that the cube superlattice is composed of pure Pt in Fig. S6.

In order to get more solid proofs that nanocrystal shape transformation induced self-assembly geometry change, we performed in-situ observation at high resolution at a dose rate of $2520 \mathrm{e} \AA^{-2} \mathrm{~s}^{-1}$. With higher dose rate we could get atomic resolution here, and the growth pathway and self-assembly behavior were observed from Movie S7. We found that the particle growth still followed the same trend and had a cube shape at the end. It provided direct insight on the shape transformation from the truncated octahedral to cube, which consequently induced hexagonal to square phase transition with high spatial resolution. Sequential TEM images in Fig. S7a extracted from Movie S7 show the hexagonal packed nanocrystals (marked from 0-6) transform to square packed particles. The detailed flowchart for hexagonal-to-square phase transition in Fig. S7b and the statistics of the angles' change in Fig. $S 8$ could further verify the transformation from hexagonal-to-square packed nanocrystals observed in a former experiment of low dose rate. The high resolution TEM (HRTEM) images in Fig. 5a show the individual nanocrystals' shape evolution. These atomic resolution images depict the facet development of nano- 
a
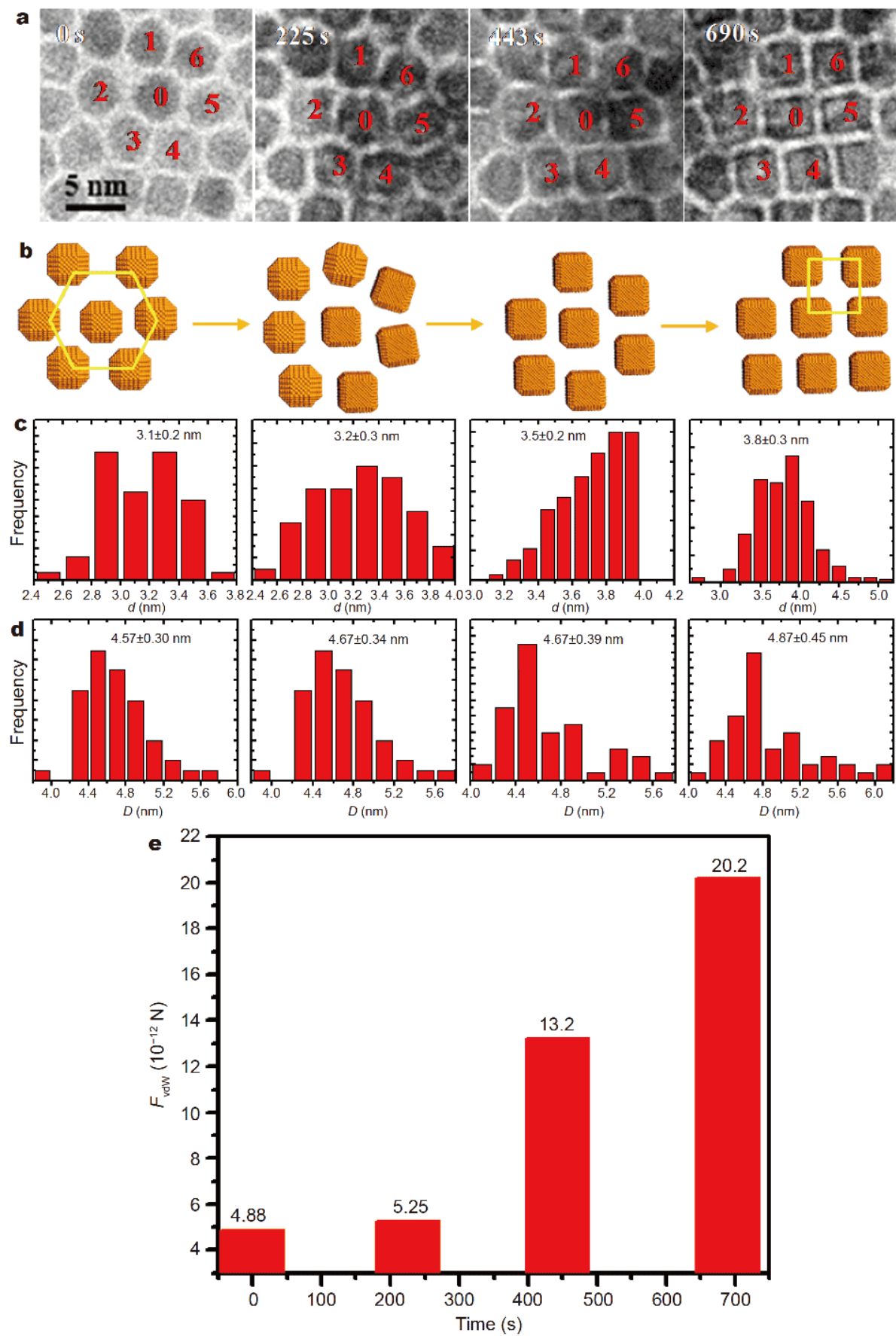

Figure 4 In-situ TEM observation of the hexagonal-to-square phase transition in a large superlattice. (a) Sequential TEM images showing the local phase transition from hexagonal to square in a large superlattice extracted from Movie S6; (b) the detailed flowchart for the hexagonal-to-square phase transition process; (c) the quantitative particle size distribution ( $d$ is the diameter of nanoparticle) on the temporal evolution of the shape of nanocrystals in a large superlattice; (d) the quantitative nanoparticle center-to-center distance $D$ on the temporal evolution of the shape of nanocrystals in a large superlattice; (e) $F_{\mathrm{vdW}}$ analysis during the hexagonal-to-square phase transition process.

crystal and demonstrate clearly the growth process from truncated octahedron to nanocube [30]. Simulated TEM images of the Pt nanoparticle in Fig. 5b further confirm the shape evolution. Moreover, Movie S8 provides more details on the transition process from the hexagonal to square phase at a high spatial resolution. As shown in 


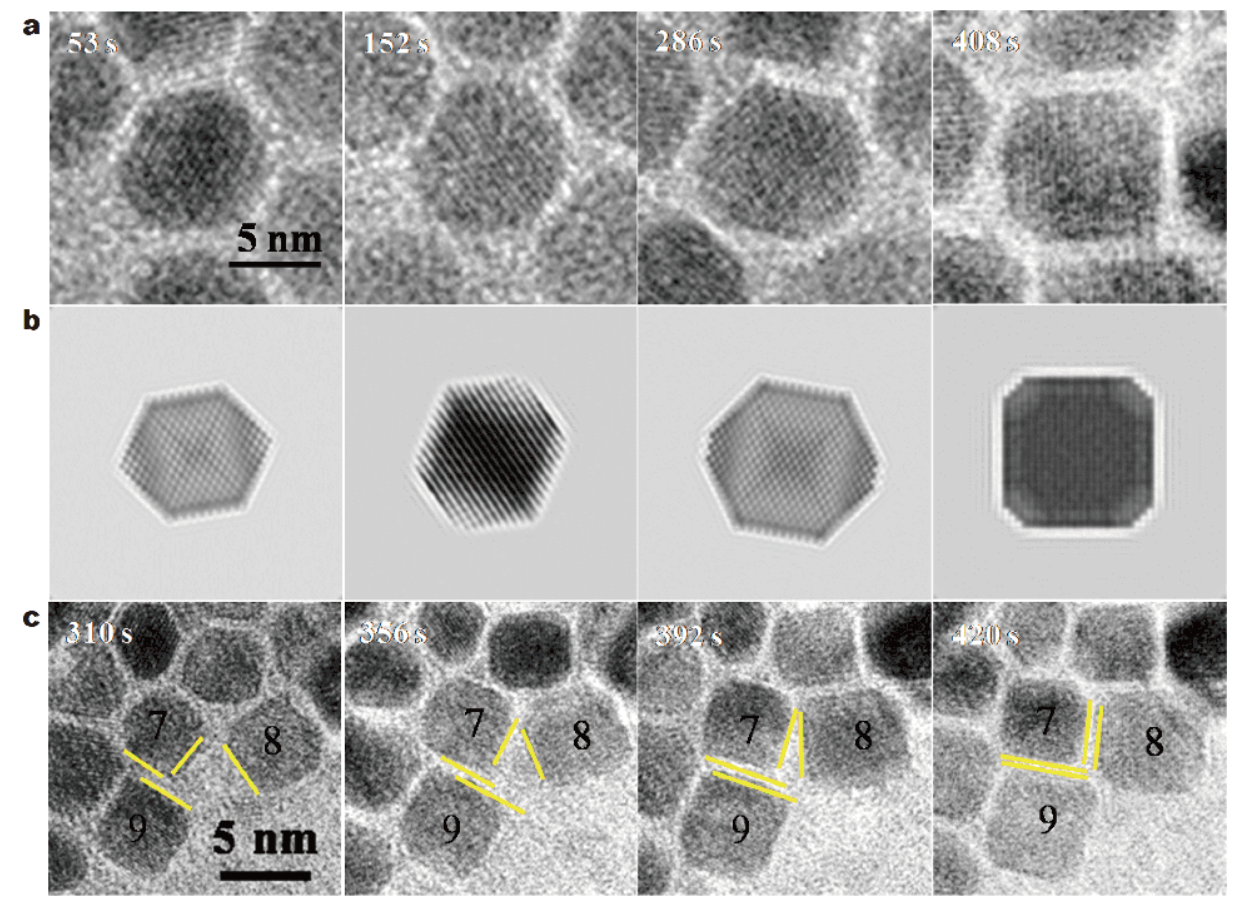

d
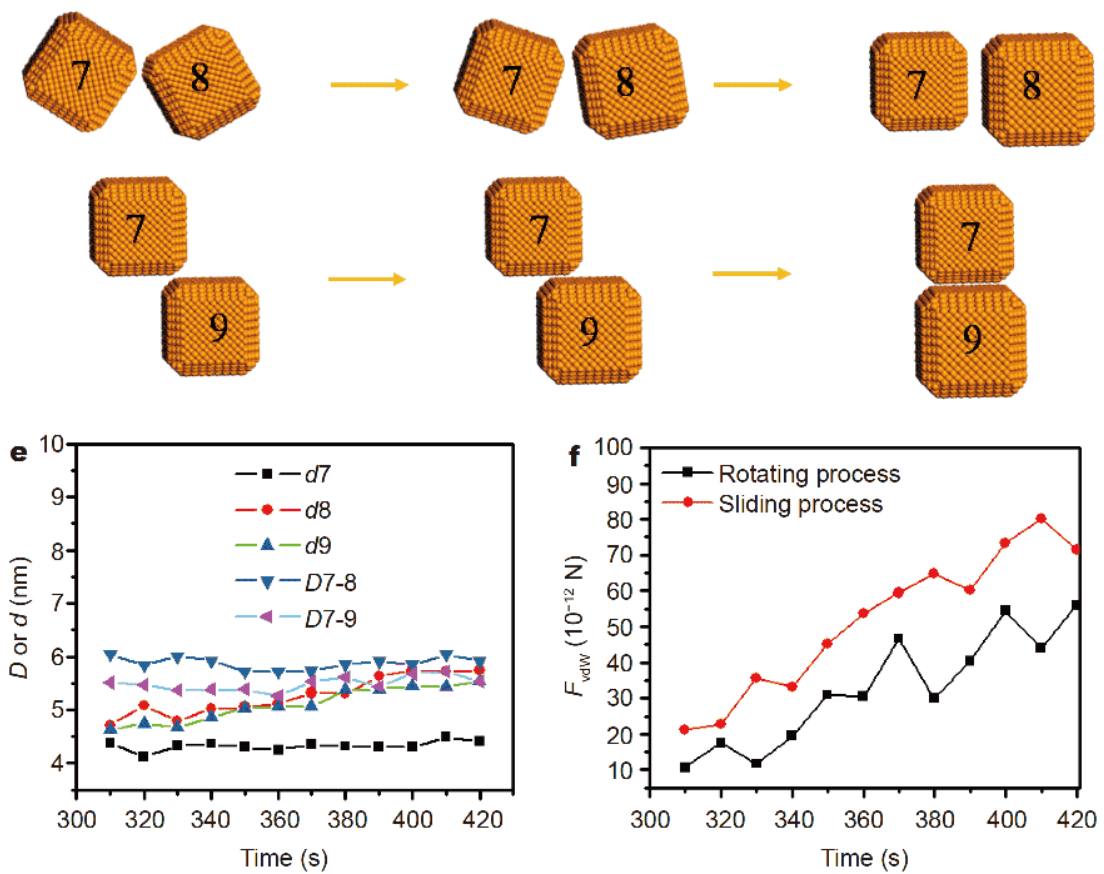

Figure 5 In-situ HRTEM observation of facet development and the self-assembly process of the cube pairs in the liquid cell. (a) Sequential HRTEM images showing the facet development of the Pt nanocube extracted from Movie S7; (b) simulated HRTEM images of the Pt nanoparticle in (a); (c) sequential TEM images showing the self-align process of two cubes via rotating (marked by number 7 and 8 ) and sliding (marked by number 7 and 9 ) methods extracted from Movie S7; (d) the detailed flowcharts showing the rotating and sliding processes of cube pairs, respectively; (e) the diameter $d$ and center-to-center distance $D$ on the temporal evolution during the rotating and sliding processes; (f) $F_{\mathrm{vdW}}$ analyses of two cubes during the processes.

Fig. S9, at the beginning of cube formation, the nanocrystals still kept a pseudo-hexagonal geometry (4 s). In the initial stage, the nanocubes aligned with one of their [100] directions quickly. The van der Waals interactions 
between the two cubes with opposed (100) facets led to the alignment and reduced rotation freedom. At the later stage, the nanocubes beside the well aligned chain moved slowly and aligned in another [100] direction. The strong van der Waals interactions between the vertical (100) facets $(253 \mathrm{~s})$ in $1 \mathrm{~nm}$ range could maximize the particleparticle interaction and lower the whole system energy. Finally, the directionality between cube-cube in-plane $\{100\}$ attractions changed the symmetry of the superlattice from hexagonal to square $(358 \mathrm{~s})$. The gradual evolution of 2D nanocube superlattice from hexagonal to square phase was marked with red circles.

HRTEM images in Fig. $5 \mathrm{c}$ depicted the detail selfassembly process of the cube pairs. The cube pairs with random attachment could have two kinds of mismatch, one is orientation mismatch (cubes 7 and 8), and the other is position mismatch (cubes 7 and 9). As shown in Fig. 5c, a face-to-face self-assembly process of cubes 7 and 8 started from a tip to tip attachment. They attached by the corner of the cubes, then the van der Waals interactions between the vertical (100) facets drove cube 8 to rotate clockwise and finally have a parallel configuration. The statistic of angle change of two adjacent cubes in Fig. S10 depicts a slow rotation at the early stage, and then the acceleration in the rotation speed. But before the particle got fully attached, it slowed down again. Small close interact surface area at the early stage might be the reason for slow rotation. As the rotation continued, the contact area increased, and van der Waals interactions might become stronger and drove the cube to rotate faster. At the end, before it was fully stuck together, the rotation was slowdown, which may be caused by the narrow gap formation and squeeze out of DMF solvent between the two (100) facets. Accordingly, the real-time $F_{\mathrm{vdW}}$ on two cubes was calculated as shown in Fig. $5 \mathrm{f}$ (black curve), where the $F_{\mathrm{vdW}}$ increased from $1 \times 10^{-11}$ to $4 \times 10^{-11} \mathrm{~N}$ during the rotating process.

Fig. $5 \mathrm{c}$ also shows that a particle-particle sliding process started from an edge-to-edge attachment for cubes 7 and 9. The two cubes had a big position mismatch at the beginning and then cube 9 slid from the right side to left side along the $\{100\}$ facet of cube 7 . Overlap of two $\{100\}$ facets on cubes 7 and 9 continued to increase and finally had a nearly prefect alignment and maximum surface area overlap. The statistic of overlap distance change of two adjacent cubes in Fig. S11 confirms the sliding process. Accordingly, the real-time $F_{\mathrm{vdW}}$ on two cubes was calculated as shown in Fig. $5 \mathrm{f}$ (red curve), where the $F_{\mathrm{vdw}}$ increased from $2 \times 10^{-11}$ to $6 \times 10^{-11} \mathrm{~N}$ during the sliding process, clearly depicting the sliding process was driven by $F_{\mathrm{vdW}}$ and such a facets overlap of cubes between the vertical $\{100\}$ facets can keep the maximum van der Waals interactions. The schematics in Fig. 5d depicts the detail rotating and sliding processes, respectively.

The influence of electron beam was considered. We have observed the hexagonal-to-square phase transition

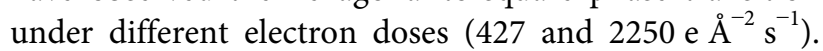
Additionally, Fig. S12 and Movie S9 show the nanoparticle coalescence and overgrowth are captured at $3320 \mathrm{e} \AA^{-2} \mathrm{~s}^{-1}$. It is noted that the cube and ordered superlattice are not formed. The high electron beam dose and long-time electron illumination resulted in the removal of the surface ligand of nanoparticles and induced the nanoparticle coalescence and overgrowth [28]. The stability of square geometry was also evaluated in Fig. S13 and Movie S10, where the square geometry was relatively

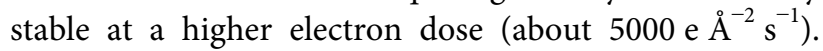
Furthermore, we also provided some results about the assembling process of pre-synthesized Pt cube by ex-situ TEM. First, Pt nanocubes were synthesized in Pt salt precursor solution through a solvothermal method according to previous work [29]. The TEM image of the nanocrystals in Fig. 6a clearly shows the cube morphology. The nanocubes attach face-to-face by $\{100\}$ facets with almost no mismatch, thus producing a square geometry. The HRTEM image of nanocube in Fig. $6 \mathrm{~b}$ depicts the lattice fringe spacing of cube is $0.20 \mathrm{~nm}$. So we could clearly identify the flat $\{100\}$ surface of all nanocrystal facets. High angle annular dark-field scanning TEM (HAADF-STEM) image illustrates the self-assembled nanocubes in Fig. 6c, which depicts the well alignment of nanocubes along [100] orientation. Energy-dispersive Xray spectroscopy (EDS) mapping image in Fig. 6d further confirms that Pt nanocubes are the dominant products. We can accordingly draw a conclusion that the shape evolution of nanocubes and the van der Waals interactions between the vertical (100) facets of cube play a key role in the phase transformation from the hexagonal to square geometry.

\section{CONCLUSIONS}

In conclusion, we have visualized that the particle shape change induces local phase transition of Pt nanocrystal superlattice from hexagonal to square configuration by in-situ liquid cell TEM. Our observations also reveal that the long-range ordering hexagonal superlattice could form either by shrinking and reordering of nanocrystal aggregate or single nanoparticle attachment at the kink site. As the nanocrystals' shape transformed from truncated octahedron to cube, the local phase transition from 


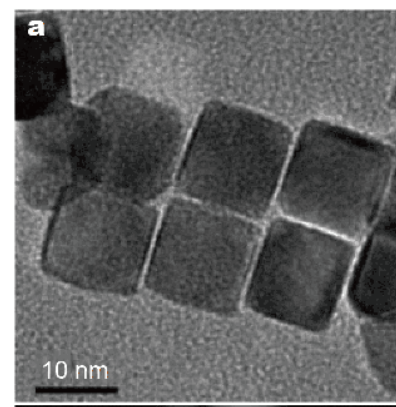

b

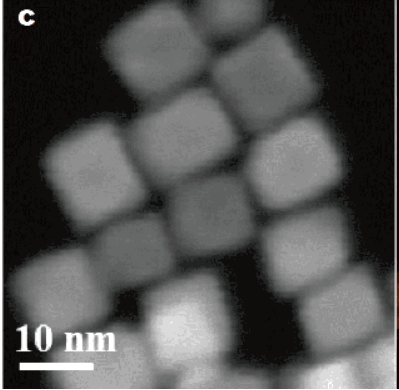

d

Figure 6 TEM (a), HRTEM (b), HADDF-STEM (c) and EDS mapping (d) images of Pt nanocrystals prepared from $\mathrm{Pt}(\mathrm{acac})_{2}$ precursor in $\mathrm{DMF}$ solution.

hexagonal-to-square ordering was achieved by collective particles sliding and rotation. The quantitative analysis of the van der Waals force between nanocrystals depicted that the transition from hexagonal to square symmetry induced the increase of van der Waals force between cubes, which was the main driving force for phase transition and the origin of $2 \mathrm{D}$ superlattice stabilization. It is clear that the transition from hexagonal to square symmetry depends sensitively on the nanocrystal shape, which also determines the directionality of the attractive interaction. Moreover, the ability to observe these dynamic processes in real time and real space could provide kinetic information about self-assembly, which can predict and direct the self-organization of nanostructures.

Received 25 September 2019; accepted 13 November 2019; published online 12 December 2019

1 Tao A, Sinsermsuksakul P, Yang P. Tunable plasmonic lattices of silver nanocrystals. Nat Nanotech, 2007, 2: 435-440

2 Stebe KJ, Lewandowski E, Ghosh M. Oriented assembly of metamaterials. Science, 2009, 325: 159-160

3 Ghosh SK, Pal T. Interparticle coupling effect on the surface plasmon resonance of gold nanoparticles: From theory to applications. Chem Rev, 2007, 107: 4797-4862

4 Shen H, Chen L, Ferrari L, et al. Optical observation of plasmonic nonlocal effects in a 2D superlattice of ultrasmall gold nanoparticles. Nano Lett, 2017, 17: 2234-2239

5 Zaluzhnyy IA, Kurta RP, André A, et al. Quantifying angular correlations between the atomic lattice and the superlattice of nanocrystals assembled with directional linking. Nano Lett, 2017,
17: $3511-3517$

6 Ye X, Chen J, Eric Irrgang M, et al. Quasicrystalline nanocrystal superlattice with partial matching rules. Nat Mater, 2017, 16: 214219

7 Lee YH, Shi W, Lee HK, et al. Nanoscale surface chemistry directs the tunable assembly of silver octahedra into three two-dimensional plasmonic superlattices. Nat Commun, 2015, 6: 6990

8 Henzie J, Grünwald M, Widmer-Cooper A, et al. Self-assembly of uniform polyhedral silver nanocrystals into densest packings and exotic superlattices. Nat Mater, 2011, 11: 131-137

9 Gong J, Newman RS, Engel M, et al. Shape-dependent ordering of gold nanocrystals into large-scale superlattices. Nat Commun, 2017, 8: 14038

10 Zhuang Z, Peng Q, Zhang B, et al. Controllable synthesis of $\mathrm{Cu}_{2} \mathrm{~S}$ nanocrystals and their assembly into a superlattice. J Am Chem Soc, 2008, 130: 10482-10483

11 Jones MR, Osberg KD, Macfarlane RJ, et al. Templated techniques for the synthesis and assembly of plasmonic nanostructures. Chem Rev, 2011, 111: 3736-3827

12 Josten E, Wetterskog E, Glavic A, et al. Superlattice growth and rearrangement during evaporation-induced nanoparticle selfassembly. Sci Rep, 2017, 7: 2802

13 Bein B, Hsing HC, Callori SJ, et al. In situ X-ray diffraction and the evolution of polarization during the growth of ferroelectric superlattices. Nat Commun, 2015, 6: 10136

14 Terán Arce F, Vela ME, Salvarezza RC, et al. Dynamic characteristics of adsorbed monolayers of 1-dodecanethiol on gold (111) terraces from in-situ scanning tunneling microscopy imaging. Electrochim Acta, 1998, 44: 1053-1067

15 Weidman MC, Smilgies DM, Tisdale WA. Kinetics of the selfassembly of nanocrystal superlattices measured by real-time in situ X-ray scattering. Nat Mater, 2016, 15: 775-781

16 Liao HG, Zheng H. Liquid cell transmission electron microscopy. Annu Rev Phys Chem, 2016, 67: 719-747

17 Zeng Z, Zheng W, Zheng H. Visualization of colloidal nanocrystal formation and electrode-electrolyte interfaces in liquids using TEM. Acc Chem Res, 2017, 50: 1808-1817

18 Ross FM. Opportunities and challenges in liquid cell electron microscopy. Science, 2015, 350: aaa9886

19 Jeong M, Yuk JM, Lee JY. Observation of surface atoms during platinum nanocrystal growth by monomer attachment. Chem Mater, 2015, 27: 3200-3202

20 Li D, Nielsen MH, Lee JRI, et al. Direction-specific interactions control crystal growth by oriented attachment. Science, 2012, 336: 1014-1018

21 Aabdin Z, Lu J, Zhu X, et al. Bonding pathways of gold nanocrystals in solution. Nano Lett, 2014, 14: 6639-6643

22 Park J, Zheng H, Lee WC, et al. Direct observation of nanoparticle superlattice formation by using liquid cell transmission electron microscopy. ACS Nano, 2012, 6: 2078-2085

23 Lee WC, Kim BH, Choi S, et al. Liquid cell electron microscopy of nanoparticle self-assembly driven by solvent drying. J Phys Chem Lett, 2017, 8: 647-654

24 Kim J, Jones MR, Ou Z, et al. In situ electron microscopy imaging and quantitative structural modulation of nanoparticle superlattices. ACS Nano, 2016, 10: 9801-9808

25 Powers AS, Liao HG, Raja SN, et al. Tracking nanoparticle diffusion and interaction during self-assembly in a liquid cell. Nano Lett, 2017, 17: 15-20

26 Lee J, Nakouzi E, Song M, et al. Mechanistic understanding of the 
growth kinetics and dynamics of nanoparticle superlattices by coupling interparticle forces from real-time measurements. ACS Nano, 2018, 12: 12778-12787

27 Wang Y, Peng X, Abelson A, et al. Dynamic deformability of individual $\mathrm{PbSe}$ nanocrystals during superlattice phase transitions. Sci Adv, 2019, 5: eaaw5623

28 Wang Y, Peng X, Abelson A, et al. In situ TEM observation of neck formation during oriented attachment of PbSe nanocrystals. Nano Res, 2019, 12: 2549-2553

29 Gumeci C, Marathe A, Behrens RL, et al. Solvothermal synthesis and electrochemical characterization of shape-controlled Pt nanocrystals. J Phys Chem C, 2014, 118: 14433-14440

30 Liao HG, Zherebetskyy D, Xin H, et al. Facet development during platinum nanocube growth. Science, 2014, 345: 916-919

Acknowledgements This work was financially supported by the National Key Research and Development Program of China (2017YFA0206500), and the National Natural Science Foundation of China (21673198, 21373008 and 21621091).

Author contributions Liao HG conceived the study and guided the whole project. Zhang J designed and performed the experiments. Zhang J, Liao HG and Sun SG participated in data analysis and wrote the manuscript. All authors contributed to the general discussion.

Conflict of interest The authors declare that they have no conflict of interest.

Supplementary information Supporting data are available in the online version of the paper.

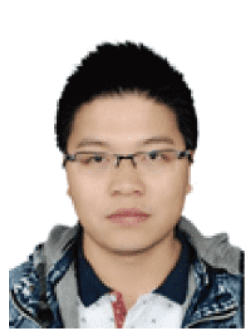

Junyu Zhang is currently a $\mathrm{PhD}$ student under the supervision of Profs Shi-Gang Sun and Hong-Gang Liao at the College of Chemistry and Chemical Engineering, Xiamen University, China. He received his BSc degree in 2011 from Fujian Agriculture and Forestry University and Master degree in 2015 from Fuzhou University, respectively. Recently, his research interests include the assembly of hybrid semiconductors and metal-based novel energy materials for photoelectrochemical applications and in-situ TEM techniques.

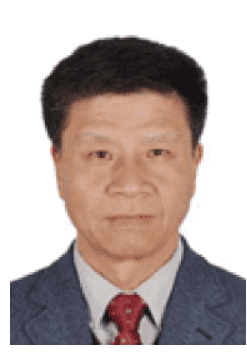

Shi-Gang Sun is a professor at the State Key Laboratory of Physical Chemistry of Solid Surfaces, College of Chemistry and Chemical Engineering, Xiamen University, China. $\mathrm{He}$ received his BSc degree of chemistry in 1982 from Xiamen University and $\mathrm{PhD}$ at d'Etat in 1986 from Universite' Pierre et Marie Curie (ParisVI) and carried out post-doctoral research at the Laboratoire d'Elec-trochimie Interfaciale du CNRS, France. He is currently a Fellow of the Royal Society of Chemistry, UK, and a Fellow of the International Society of Electrochemistry. His research interests include electrocatalysis and electrochemical surface science.

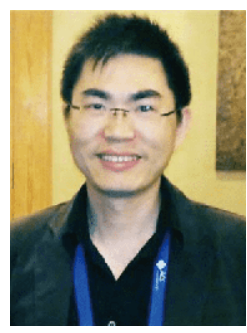

Hong-Gang Liao received his $\mathrm{PhD}$ from Xiamen University. He worked as a Research Associate in Lawrence Berkeley National Laboratory since 2011. Then he got the "Thousand Talents Plan" in China and became a professor in Xiamen University in 2015. His research focuses on imaging through liquids to address fundamental materials issues in colloidal synthesis, energy conversion and energy storage application by in situ liquid cell TEM.

\section{Pt超晶格组装和对称性转化的原位液体TEM研究}

张俊玉, 孙世刚, 廖洪钢 ${ }^{*}$

摘要 具有精确控制结构的二维 $(2 \mathrm{D})$ 纳米晶超晶格在光子、等离 子体和光电子应用中具有重要意义, 并已被广泛研究, 但在理解超 晶格的形成机理和发展途径方面仍然存在挑战. 本文采用液体池 透射电镜技术原位观察了铂二维超晶格的形成和六配位到四配位 的局部相转化过程. 胶体纳米晶在溶液中流动时, 通过纳米晶的收 缩和重排或者纳米晶的附着形成长程有序的六配位超晶格. 当纳 米晶的形貌由截角八面体转变为立方体时, 六配位超晶格重新排 列为四配位立方超晶格. 此外, 我们的观察和定量分析结果表明, 从六配位到四配位的相变主要是由垂直 $\{100\}$ 面之间的强范德华相 互作用引起的. 实时追踪 $2 \mathrm{D}$ 立方体超晶格的形成可以为超晶格组 装和稳定机制提供独特的见解. 